\title{
Transmuscular course of the medial sural cutaneous nerve and its clinical implications Sharma $S^{1}$, Sharma $T^{2}$, Bhardwaj $S^{3}$
}

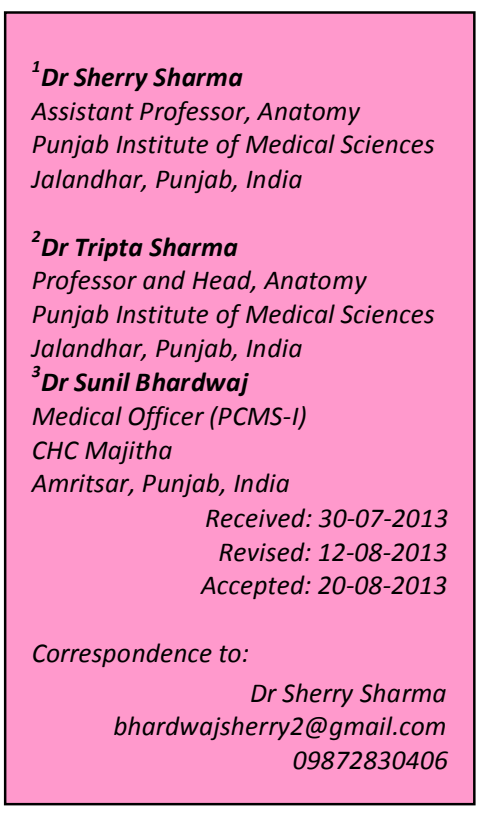

\section{Introduction}

The sural nerve is a sensory nerve that originates in lower leg and provides sensory innervations to lateral and posterior part of inferior third of leg and lateral side of foot. ${ }^{[1]}$ Classically the sural nerve comes from communication between medial cutaneous nerve of leg which is branch of tibial nerve and perforating branch from lateral cutaneous nerve which is a branch of common peroneal nerve. ${ }^{[2]}$ Sural nerve is a cutaneous nerve which begins as a branch of tibial nerve (medial sural nerve) and descends between two heads of gastrocnemius muscle, under posterior fascia which perforates to receive communicating branch from lateral cutaneous nerve of leg at different levels. The sural nerve thus formed runs slightly obliquely downwards crossing lateral border of tendo calcaneous and passing behind and then below lateral malleolus and crosses dorso-lateral aspect of foot. ${ }^{[3]}$

The anatomic course of nerve in distal leg and ankle makes it susceptible to local trauma and also increases the vulnerability to trauma during various surgical interventions. [4] Clinically, sural nerves are used widely for diagnostic purposes as in nerve conduction velocity studies and for biopsy. Since the sural nerve is the most frequently used sensory nerve in nerve transplantation, familiarity with variations in its course and distribution plays important role in these procedures. ${ }^{[5]}$ It will be appreciated from the fact that this is the main nerve used to evaluate sensory axonal loss in distal axonal neuropathies, 
primarily because sural nerve mononeuropathy is a very rare event. [6] The intactness of this nerve is also assessed when diagnosing certain peripheral neuropathies of unknown etiology. ${ }^{[7]}$ This assessment must be done carefully since alterations may be associated with symptoms such as hypoesthesia in the area innervated by the sural nerve and mild persistent pain. ${ }^{[8]}$

Although generally considered to be a sensory nerve, the existence of some communications between the sural and tibial nerves in humans suggests that the sural nerve may have a motor function. ${ }^{[9]}$ This possibility increases the clinical importance of this nerve, especially in the electrophysiological diagnosis of tibial nerve dysfunctions that affect plantar muscle innervation (tarsal tunnel syndrome). In this report, we describe one case in which the medial sural cutaneous nerve passes through the gastrocnemius muscle and discuss the clinical implications of this anatomical variation.

\section{Case Report}

During the routine educational dissections for the medical undergraduate students in Department of Anatomy, PIMS, Jalandhar, in a male cadaver fixed and preserved in $10 \%$ formalin we found an unusual transmuscular course of sural nerve in its left leg. Deep fascia from posterior surface of popliteal fossa was stripped off and fat from its upper angle was removed to expose the nerve. The length of the muscular course was $6.4 \mathrm{~cm}$. (Fig.1, 2)

The age of cadaver was approximately 50 years. This variation in the course of the medial sural cutaneous nerve was observed only in the left leg and there were no anomalies in the course of this nerve in the right leg.

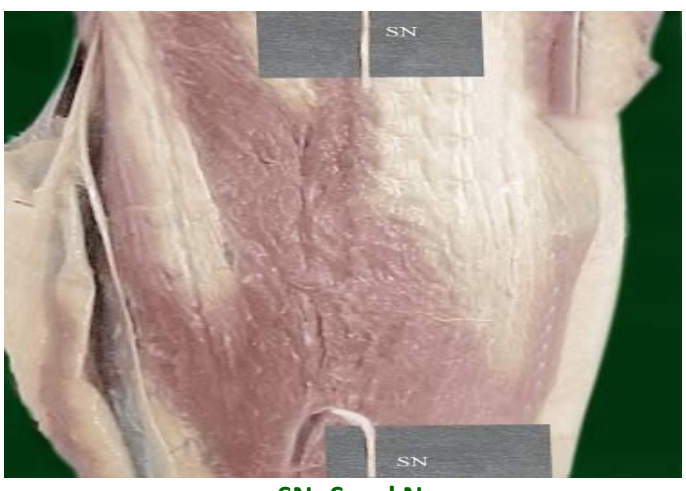

SN- Sural Nerve

Fig. 1 Showing Transmuscular Course of Medial Sural Cutaneous Nerve

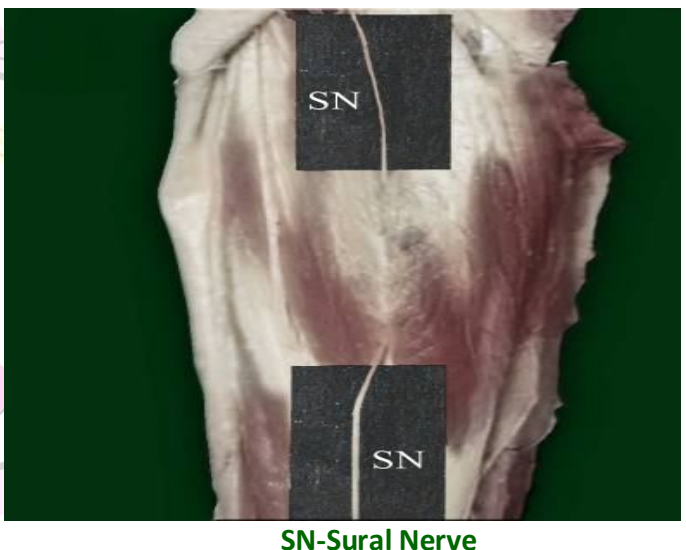

Fig.2 Showing Subfascial course of Sural Nerve

\section{Discussion}

The classification of sural nervous system explains symptomatology of its compression as it crosses posterior fascia. Sural nerve can be lacerated or compressed anywhere along its course from its exit in calf fascia to foot. Symptoms include pain, paraesthesia and sensory loss over lateral aspect of foot and ankle corresponding to sural nerve territory. [10] Prolonged compression, calcaneal tendon reconstructive procedures or direct trauma associated with fractures may lead to sural 
neuropathies anywhere along the course of the nerve. ${ }^{[11]}$ Sural nerve entrapment may be associated with ganglia or posttraumatic scarring. ${ }^{[12]}$ Birbilis et al ${ }^{[13]}$ reported a case of sural nerve neuropathy in which the nerve was compressed by induration of the surrounding soft tissue as a result of external pressure produced by the use of tightly laced boots. Seror [6] reported a similar finding in three males who used new ski boots; in these cases, the hypoesthesia in the external border of the right foot indicated sural nerve involvement. Husson et al ${ }^{[14]}$ described a new canal syndrome that involved increased pain in the region of the sural nerve during plantar flexion of the leg. Pain was felt because there was compression of the sural nerve by myositis ossificans when the nerve passed through a non-extensible tunnel formed by the fold of the posterior sural aponeurosis. Complete blockade of sensory conduction at the site where the sural nerve penetrates the deep fascia, with a mild imprint of the nerve, was described by Seror. ${ }^{[6]}$ In this case, the fibrosis of the deep fascia was probably related to the lower limb injury sustained ten months earlier. Bryan et al ${ }^{[3]}$ reported the case of a patient who suffered injury to the right medial gastrocnemius muscle that was complicated by referred pain in the lower leg and foot in the area innervated by the sural nerve; this pain was accentuated by plantar flexion. Surgical exploration under local anesthesia found that the sural nerve was encased in scar tissue beneath the deep fascia at the level of the gastrocnemius muscle. Coert and Dellon ${ }^{[15]}$ postulated a mechanism for entrapment of the lateral sural cutaneous nerve when it pierces the deep fascia. These authors concluded that during knee extension the fascia could exert a compressive force on the nerve, and that a biopsy of the sural nerve could result in a false diagnosis of axonal polyneuropathy. As shown by the foregoing literature reports, there are many cases in which the sural nerve or its branches are surrounded by fascia or scar tissue. However, the penetration of muscle by this nerve has been poorly documented. Maria et al ${ }^{[16]}$ has described in his case report four out of sixty dissections in which sural nerve traversed intramuscular course.

This variation is an important surgical consideration when this nerve is used as an autograft for peripheral nerve reconstruction. During the regular dissection, a precise assessment of the frequency of this muscular course is important because of the possibility of this nerve being confused with included fascia instead of the muscular course. Clinically, this muscular course of the sural nerve could account for the calf pains experienced by patients during contraction of the gastrocnemius that is responsible for plantar flexion of the leg. Clinical confirmation of this situation might include a positive Tinel's sign at the site of nerve entrapment and the electrodiagnosis of complete sensory conduction blockade. ${ }^{[16]}$ Anatomic studies have demonstrated presence of potential sites for entrapment of sural nerve as its travels through deep fascia, particularly, with knee extension which may be clinically relevant especially in patients with history of diabetes. [4] Anatomically, this variation in nerve course may be important for evaluating sensory axonal loss in distal axonal neuropathies 
since sural nerve neuropathy is a rare event. Finally, this variation should be borne in mind by clinicians, surgeons and academicians who manipulate this particular anatomical site.

\section{References}

1. Mahakkanukraun $\mathrm{P}$, Chomsung $\mathrm{R}$. Anatomical variation of sural nerve. Clin Anat 2000;15:263-266.

2. Mestdagh $H$, Drizenko A, Maynou C, Demondion $\mathrm{X}$, Monier R. Origin and makeup of the human sural nerve. Surg Radiol Anat 2001;23:307- 312.

3. Bryan BM, Lutz GE, O' Brien SJ. Sural nerve entrapment after injury to gastrocnemius: a case report. Arch Phys Med Rehabil 1999;80:604-606.

4. Stickler DE, Morley KN, Massey EW. Sural neuropathy: etiologies and predisposing factor. Muscle Nerve 2006; 34:482-484.

5. Nayak SB. Sural nerve and short saphenous vein entrapment - a case report. Ind J Plast Surg 2005;38(2):171172.

6. Seror P. Sural nerve lesions: a report of 20 cases. Am J Phys Med Rehabil 2002; 81:876-880.

7. Neundorfer B, Grahmann F, Engelhardt A, Harte U. Postoperative effects and value of sural nerve biopsies: a retrospective study. Eur Neurol 1990; 30:350-352.

8. Flachenecker $\mathrm{P}$, Janka $\mathrm{M}$, Goldbrunner R, Toyka KV. Clinical outcome of sural nerve biopsy: a retrospective study. J Neurol 1999;246:93-96.

IJMDS • www.ijmds.org • January 2014; 3(1)
9. Sekiya S, Kumaki K. Sural-tibial communication in humans. Anat Sc Int 2002;77:140-144.

10. Oh SJ, Meyer RD. Entrapment neuropathies of the tibial (posterior tibial) nerve. Neurol Clin 1999;17(3): 593-615.

11. Reisin R, Pardal A, Ruggieri V, Gold L. Sural neuropathy due to external pressure: report of three cases. Neurology1994;44:2408-2409.

12. Pringle RM, Protheroe K, Mukherjee SK. Entrapment neuropathy of the sural nerve. J Bone Joint Surg $\mathrm{Br}$ 1974;56: 465-468.

13. Birbilis TH, Ludwig HC, Markakis E. Neuropathy of the sural nerve caused by external pressure. Acta Neurochir (Wien) 2000;142:951-952.

14. Husson JL, Mathieu M, Briand $B$, Meadeb J, Barumbi O, Masse A. Syndrome of compression of the external saphenous nerve (or the sural nerve). Acta Orthop Belg 1989;55:491497.

15. Coert JH, Dellon AL. Clinical implications of the surgical anatomy of the sural nerve. Plast Reconstr Surg 1994;94: 850855.

16. Maria Lucia Pimentel, Rodrigo Mota Pacheco Fernandes, Marcio Antonio Babinski. Anomalous course of the medial sural cutaneous nerve and its clinical implications. Braz J morphol Sci 2005;22(3):179-182.

Cite this article as: Sharma S, Sharma T, Bhardwaj S. Transmuscular course of the medial sural cutaneous nerve and its clinical implications. Int J Med and Dent Sci 2014; 3(1):348-351.

Source of Support: Nil Conflict of Interest: No 